\title{
RNA sequencing reveals secrets of skin aging
}

As we age, so does our skin. One of the factors that affects how quickly skin appears to age is exposure to UV light. Dr Jeong-Sun Seo of Seoul National University Bundang Hospital, Republic of Korea, is investigating how UV light affects skin aging at the genetic level. Using Rf sequencing daV ligh Dr Seo and his team demonstrate that some signs to over-exposure to UV light.

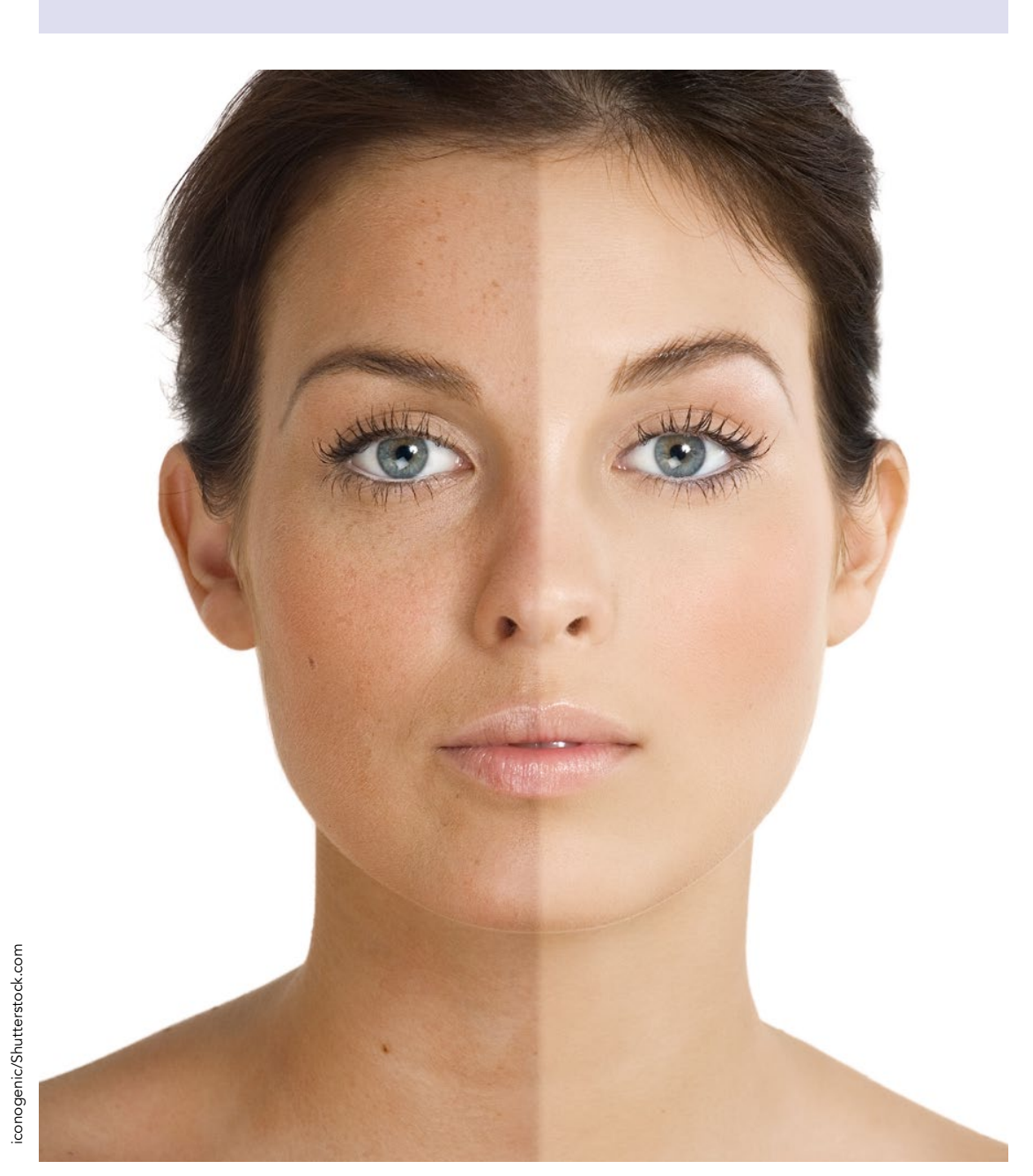

ur skin has to tolerate a great deal. Exposure to sun, wind ogging cosmetics, a poor diet; these are a few of the challenges our skin might endure throughout a lifetime. Our skin apperants for a large part of our where the most obvious signs of aging can be seen. Our skin condition reflects our apparent age; no wonder, then, that skin care is such a huge market.

Many factors are known to affect the way that skin ages. Some of these factors, such as genetic qualities, are intrinsic to the body while others are extrinsic. Of all the extrinsic factors, perhaps the one that has received the most publicity is excessive exposure to sunlight. The light, which is also the type of light used

\section{Skin offers a} unique opportunity to investigate the effects of UV light on aging.

in tanning beds and lamps. Exposure to UV light is not only an important factor in skin aging. excessive exposure to $U V$ also increases the risk of skin cancer. The dangers of sunbumn and the importance of sunscreen are well-known, but the are still under investigation.

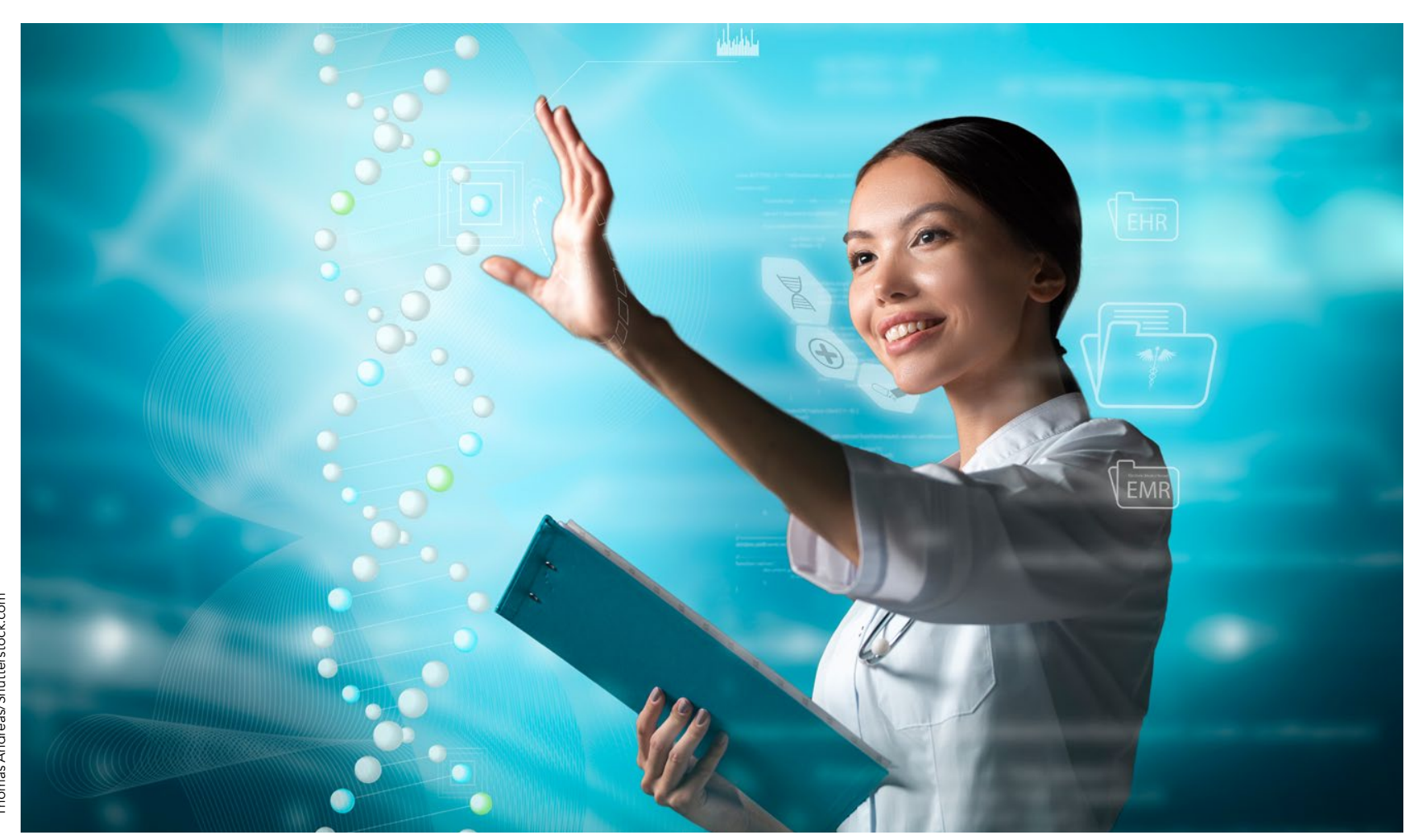

UV AND SKIN AGING

shown that UV light affects the aging process of skin by causing changes in gene expression (when and how a gene produces a protein). Exactly how this happens, however, is not well understood. Using state-of-the-art technology, Dr Seo and his team can explore genetic information to investigate how skin ages, with the aim of reaching a better understanding of this process. In particular, the team looked at a process known as photo-aging.

\section{ANALYSING THE TRANSCRIPTOME} research on the ffect of UV light on skin aging, it has focussed on the behaviour of just a few genes. While this is useful, genes are known to interact with one another in myriad ways. Focusing on a few individual genes therefore risks missing important genetic pathways that could influence the aging process. To avoid this problem, Dr Seo utilised a much broader type of genetic information to investigate gen expression changes in skin. Ribonucleic acid (RNA) is produced from DNA during the process of protein synthesis The comple set of RNA transcripts produced by the genome of a partic species - in this case, humans - is the transcriptome therefore allows earchers to characterise the genetic activity of a tissue, such as skin, as a whole; it shows which genes and which are not.

SKIN CHANGES AND UV EXPOSURE Skin offers a unique opportunity to investigate the effects of UV light on aging. Some parts of our skin are frequently exposed to sunlight, while other parts are usually kept covered. In his study, Dr Seo and his colleagues lomer leg with UV the suprapubic region (the area betwe the navel and the pubic bone).

Dr Seo's team used transcriptomic RNA sequencing data to examine the behaviour of a very large number of different genes at the same time. In total, the researchers assessed genetic data from almost 600 samples of skin. This in-depth investigation allowed the team to pin-point the actual biological changes caused by exposure of skin to UV light. Their results showed a clear difference between the transcriptomes of the lower leg and suprapubic skin samples, suggesting that gene activity is notably differentin these two areas of skin. This in expce could be due to

\section{ANGIOGENESIS AND}

Wrinkles are FrMATION sign of aging in skin. Angiogenesis - the formation of new blood vessels - is known to play a significant role in the development of wrinkles. In this study genes involved in angiogenesis were photo-danaged (nine active) in phos

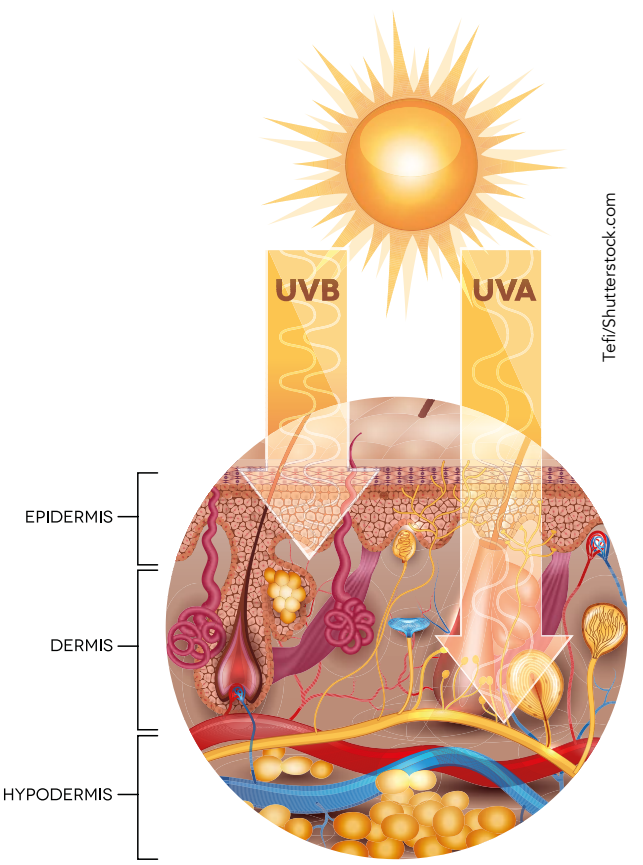


a significant difference in the activity of leg, when compared with skin from the suprapubic region. Greater angiognesis means a higher rate of development of new blood vessels, so this result suggests that exposure to UV light directly influences wrinkle formation.

\section{UV LEAVES SKIN VULNERABLE} Lipid metabolism, the process by which fats are either made or broken down in cells, is one of the factors vital to maintaining the function of the skin as a protective barrier. When lipid metabolism is impaired, the barrier function becomes less effective, leaving the skin more valherable to damage. Dr Seo found that lipid metabolism is dramatically reduced by photo-aging, suggesting skin lacking protection. Three lipid metabolism related genes in particular (known as LPN, LPK, and SMPD3) were found to be important in this process. Similarly, water loss regulation in skin was found to decrease with age. This could adversely affect both the condition and the appearance of the skin.

As skin ages, it tends to lose elasticity, which can contribute to development of wrinkles. This is partly due to a falling amount of collagen, an important connective protein, in the skin. Dr Seo and his colleagues were able to show that genes, are up-regulated in skin damaged
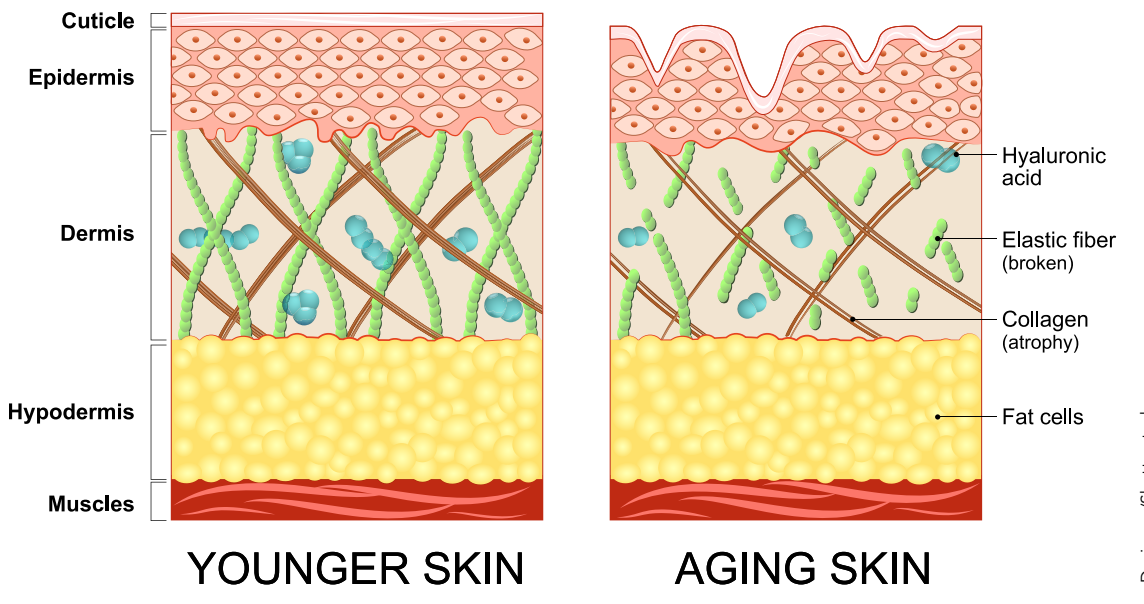

Aged skin loses elasticity com
collagen and hyaluronic acid.

Dr Seo's team used transcriptomic

RNA-sequencing data to investigate the genetic pathways of aging.

by exposure to UV light. In normal cells, older skin. Dr Seo found that the rate of MMP genes are known to play a role in breaking down collagen. When MMP genes become more active, collagen is broken down faster, leading to reduced elasticity in the skin.

\section{AGE-RELATED SKIN CHANGES} Some skin changes occur with aging whether or not the skin has been exposed to excessive sunlight. Previous work has shown that wound healing can

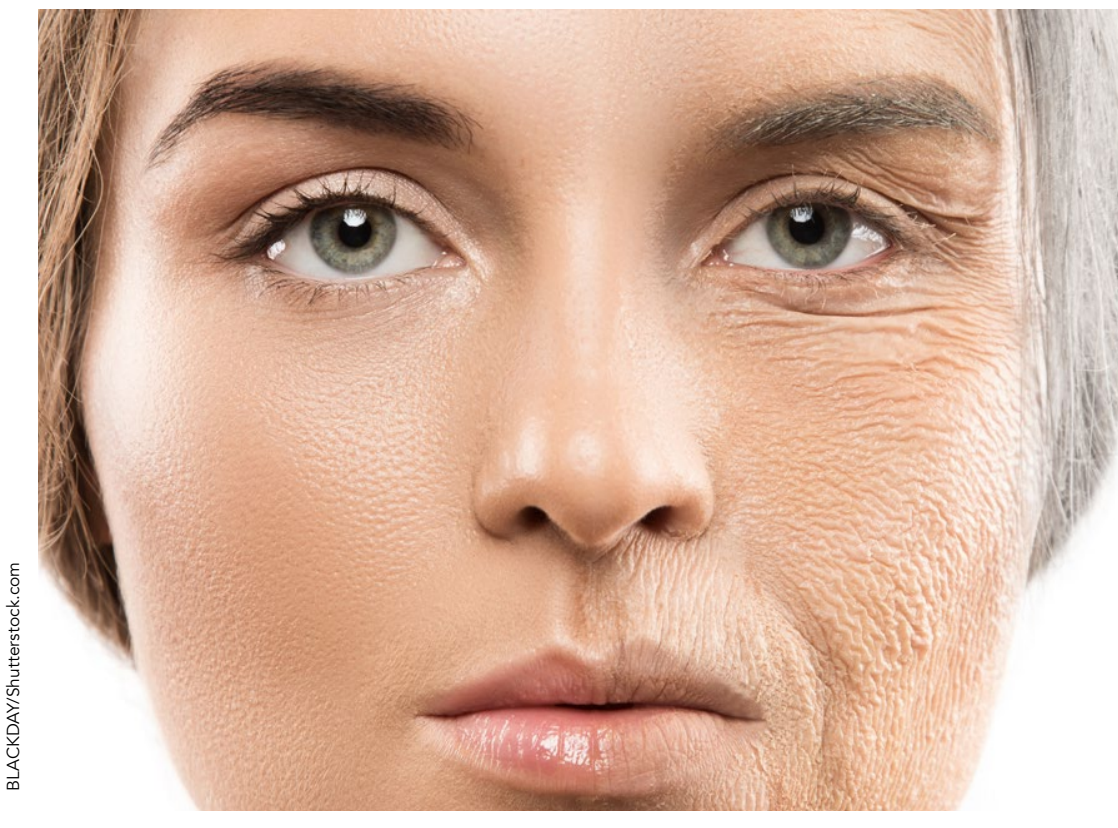
wound healing decreased with aging in both types of skin. A set of genes know to be involved in wound healing were down-regulated (became less active) in This UV exposed and UV-protected skin. This result suggests that slower healing is an inevitable consequence of aging, whether skin has been.

THE FUTURE

In many parts of the world, life expectancies have been steadily research interest in the vared increased of aging. In the field of skin aging research, Dr Seo's team is the first to use transcriptomic RNA-sequencing data to investigate the genetic pathways of aging. Photo-aging due to excessive exposure to sunlight can only be studied in the skin; it is therefore crucial that the consequences of photo-damaged skin are properly understood. This work is an important step in that direction.

In future, the knowledge gained from innovative treatments for skin aging, ncreasing health and well-being in old a vital message, one that is well but bears repeating: wear sunscreen. Dr Seo's work could be used to develop

\section{Behind the Research}

\section{Dr Jeong-Sun Seo}

\section{Research Objectives}

A leading scientist in the field of genomics Dr Jeong-Sun in cancer and rare diseases.

\section{Detail}

Precision Medicine Center

Seoul Nati University Bundang Hospital

gi-do 13605

Republic of Korea

Bio

Dr Jeong-Sun Seo is a Distinguished Professor at Seoul National University Bundang Hospital, where he is also Director of Precision Medicine Center. He established Macrogen Inc. 22 years ago, one of the first biotechnology companies in Korea. Recently, his team built the most contiguous reference genome, AK1, using de novo assembly and phasing, taking another step forward for precision medicine in Northeast Asia.

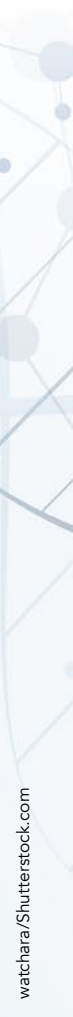

References

Cho, B, Yoo, S \& Seo, J. (2018). Signatures of photo-aging and intrinsic aging in skin were revealed by transcriptome network analysis. Aging, 10, 1-14.

\section{Personal Response}

How can transcriptomic RNA sequencing be used in other areas of aging research?

II With advanced medical care and technology. the elderly population has greatly increased compared to several decades ago. Although aging is not a transcriptome of each to predict the occurrence of diseases through assessing the levels of gene expression or use it as a tool to find a therapeutic target for each disease.
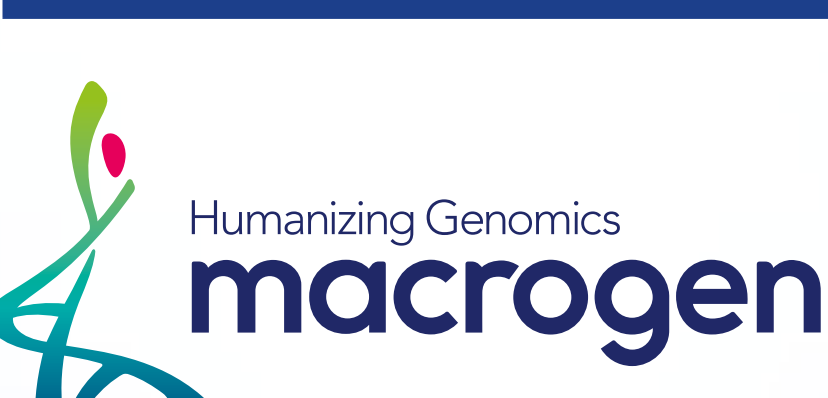